\title{
A Review on Methods for Prevention of SQL Injection Attack
}

Prof. Sweta Raut ${ }^{1}$, Akshay Nikhare*2, Yogesh Punde ${ }^{3}$, Snehal Manerao ${ }^{4}$, Shubham Choudhary ${ }^{5}$

${ }^{1}$ Assistant Professor, Computer Science and Engineering, Nagpur Institute of Technology, Nagpur, Maharashtra, India

•2,3,4,5 Students, Computer Science and Engineering, Nagpur Institute of Technology, Nagpur, Maharashtra, India

\section{ABSTRACT}

Web applications generally interact with backend information to retrieve persistent data and then present the information to the user as dynamically generated output, like HTML websites. This communication is commonly done through a low- level API by dynamically constructing query strings within a general-purpose programming language. SQL Injection Attack (SQLIA) is one of the very serious threats to web applications. This paper is a review on preventing technique for a SQL injection attack which can secure web applications against SQL implantation. This paper also demonstrates a technique for preventing SQL Injection Attack (SQLIA) using Aho-Corasick pattern matching algorithm.

Keywords : SQL Injection attack, Pattern matching, Aho-Corasick algorithm

\section{INTRODUCTION}

SQL Injection Attacks have been one of the danger's hazards for Web applications [1]. Web applications that are vulnerable against SQL blend may allow an attacker to improvement finish access to their databases. Since these databases now and again contain delicate customer information, the going with security encroachment can interweave markdown intimidation, loss of puzzle information, and twisting. Now and then, attackers can even use a SQL imbuement nonappearance of protection to dismantle control of and fall the framework that has the Web application. Web applications that are frail against SQL Injection Attacks (SQLIAs) are paying little respect to what you look like at it. To get directly to the point, SQLIAs have plausibly in view of discernible manhandled individuals, for instance, Travelocity, Ftd.com, and Surmise Inc. SQL implantation gathers a class of code-imbuement attacks in which data gave by the customer is joined in a SQL ask for in such a course, to the point that bit of the customer's information is regulated as SQL code. By utilizing these vulnerabilities, an attacker can submit SQL summons unmistakably to the database. These strikes are a certifiable peril to any Web applications that get responsibility from customers and solidify it into SQL ask for to a major database. Most Web applications used on the Web or inside huge business structures work in this manner and could thusly are defenseless against SQL imbuement. A champion among the most gainful instruments to shield against web ambushes uses Interruption Discovery System (IDS) and Network Intrusion Detection System (NIDS). An IDS uses mishandle or assortment from the standard range to ensure against attack. IDS that usage trademark assertion framework makes a gage of customary utilize outlines. Abuse perceiving affirmation reasoning uses especially known cases of unapproved provoke to presume and find happening as intended in every way that really matters unclear kind of 
strikes. These sorts of cases are called a signature. NIDS is not helping for the affiliation sorted out applications (web strike), in light of how NIDS are functioning lower level layers [2].

\section{LITERATURE SURVEY}

Beuhrer [3] has depicted a system to frustrate and to keep away from SQL blend attacks. The strategy relies upon looking, parse tree of the SQL verbalization before the union of client responsibility with the one that following after idea of duty, at run time. In another paper [3] author proposes a system to keep this sort of control and consequently dispose of SQL injection vulnerabilities. The system depends on looking at, at run time, the parse tree of the SQL proclamation before incorporation of client contribution with that subsequent after consideration of info. The solution is proficient, adding around $3 \mathrm{~ms}$ overhead to database inquiry costs. Also, it is effectively received by application software engineers, having an indistinguishable syntactic structure from current mainstream record set recovery strategies. This usage limits the exertion required by the software engineer, as it catches both the proposed inquiry and genuine question with negligible changes required by the developer, tossing a special case when fitting. This structure execution is needed to restrict the endeavor the planner needs to take; since, it in this way gets, both the true blue address and the proposed ask for and that additionally, with irrelevant changes in a general sense to be finished by the item manufacture.

Saltzer and Schroeder [4] propose a security structure against the strikes like SQL Injection. They proposed a structure utilizing unmistakable stages. One of them was the shield defaults, on which the positive destroying is poor or takes after, confers that a traditionalist course of action must be secured around wrangle about why articles ought to be open, instead of why they ought not. Data speak to today a significant resource for organizations and associations and must be secured. The vast majority of an association's delicate and exclusive data dwells in a Database Management System (DBMS). The concentration of this proposition is to create propelled security answers for ensuring the data living in a DBMS. This procedure [5] is to build up an Intrusion Detection (ID) component, actualized inside the database server that is equipped for distinguishing strange client solicitations to a DBMS. The key though is to learn profiles of clients and applications interfacing with a database. A database asks for that strays from these profiles is then named as peculiar. A noteworthy segment of this work includes prototype usage of this ID instrument in the PostgreSQL database server. Author additionally propose to enlarge the ID system with an Intrusion Response motor that is fit for issuing a proper reaction to an abnormal database ask. In a farreaching system, a couple of articles will be deficiently considered, so a default of nonappearance of consent is more secure. A layout or use mess up in an area that gives unequivocal concur tends to bomb by declining endorsement, a secured condition, since it will be immediately observed. On the other hand, a setup or use misuse in a framework that explicitly rejects get to tends to bomb by allowing get to, a mistake which may go unnoticed in standard utilize. This oversee applies both to the outward appearance of the insistence framework and to its hidden execution.

A new paper [6] introduces a scientific categorization of intrusion detection frameworks that is then used to overview and orders various research prototypes. The scientific categorization comprises an arrangement first of the detection standard, and second of certain operational parts of the intrusion 
detection framework accordingly. The frameworks are likewise assembled by the expanding trouble of the issue they endeavor to address. These groupings are utilized presciently, pointing towards various ranges of future research in the field of intrusion detection.

Yusufovna [7] has demonstrated the use of information tunneling approaches for IDS. The fast advancement of innovation and the expanded network among its segments forces new digital security challenges. To handle this developing pattern in PC attacks and react dangers, industry experts and scholastics are uniting keeping in mind the end goal to construct Intrusion Detection Systems (IDS) that join high exactness with low unpredictability and time productivity. An intrusion detection framework works by deciding if an arrangement of activities can be esteemed as an intrusion on a premise of at least one shows of intrusion. This model [7] portrays a rundown of states or activities as great or terrible (potential intrusion). These ID strategies can be actualized into two distinctive framework categorizations. Anomaly detection framework which is recognized organize movement conduct and abuse detection framework which constructs its detection in light of marks or pattern matching, likewise depicted as learning based. The display article gives a review of existing Intrusion Detection Systems (IDS) alongside their fundamental standards. Likewise, this article contends whether data mining and it's center component which is information disclosure can help in making Data mining based IDSs that can accomplish higher exactness to novel sorts of intrusion and show more powerful conduct contrasted with customary IDSs. Interruption disclosure can be named as of seeing activities that endeavor to risk the security, consistency, and transparency of the advantages of a framework. IDS show is shown and what's more its impediment in picking security infringement is displayed in this paper.

Halfond and Orso [8] had displayed an improvement for disclosure and repulsiveness of SQLIA. This technique made depended on upon the approach that ordinary to perceive the malignant demand before their execution inside the database. To accordingly produce a model of the certifiable or right request, the static piece of the strategy utilized the program examination. This could be conveyed by the application itself. The system utilized the runtime looking for an examination of proficiently made demand and to check them against the static edge appear. Halfond and Orso [9] had proposed a system for countering SQL imbuement. The framework really joined the traditionalist static examination and runtime checking for revelation and stoppage of unlawful demand before they are executed on the database. The structure gathers an immediate model of the honest to goodness request that could be made by the application in its static parts. The system assessed the dynamically made demand for consistency with statically construct appears in its dynamic part. W. G. J. Halfond ET. al. [10] proposed another, much-mechanized methodology for guaranteeing existing Web applications against SQL implantation. This technique has both handled and reasonable positive conditions over most existing structures. From the discovered perspective, the system is secured around the first idea to make sure pulverizing and the probability of vernacular structure noteworthy evaluation. From the sensible point of view, the method is the right and valuable and has irrelevant methodology necessities.

This paper [11] depicts a straightforward, effective calculation to find all events of any of a limited number of watchwords in a string of content. The 
calculation comprises of building a limited state pattern matching machine from the catchphrases and afterward utilizing the pattern matching the machine to process the content string in a solitary pass. Development of the pattern matching machine requires significant investment relative to the whole of the lengths of the catchphrases. The quantity of state advances made by the pattern matching machine in handling the content string is autonomous of the number of catchphrases. The calculation has been utilized to enhance the speed of a library bibliographic pursuit program by a factor of 5 to 10.The strategy proposed in this paper appropriate for applications in which we are searching for events of substantial quantities of watchwords in content strings. Since no extra data should be added to the content string, hunts can be made over self-assertive documents. Some data recovery frameworks figure a list or concordance for a content document to permit hunts to be led without scanning the greater part of the content string. In such frameworks rolling out improvements to the content, the document is costly in light of the fact that after each change the record to the record must be refreshed. Thus, such frameworks work best with long static content documents and short patterns.

\section{RELATED WORK}

\section{A. Types of SQL Injection Attacks}

In this area, we appear and examine the changed sorts of SQL Injection Attacks. The unmistakable sorts of strikes are everything considered not performed in the partition; a solid piece of them are utilized together or continuously, subordinate upon the particular objectives of the attacker. Note besides that there are boundless groupings of each strike sort.

\section{Tautologies}

Redundancy based attacks are among the minimum troublesome and best-known sorts of SQLIAs. The general target of a redundancy-based ambush is to mix SQL tokens that make the request prohibitive decree constantly evaluate to genuine. This system implants announcements that are always bona fide so the request reliably return comes interminable supply of WHERE condition.

Injected query: select name from user_details where username $=$ "abc" and watchword $=$ or $1=1$.

\section{Piggybacked}

In this attack, an interloper tries to inject additional inquiries close by the primary request, which are said to "piggy-back" onto the main inquiry. Along these lines, the database gets various SQL inquiries for execution additional request is added to the principal request. This ought to be conceivable by using an inquiry delimiter, for instance, ";", which deletes the table decided.

Injected Query: select name from user_details where username $=$ 'abc'; droptable acc -

\section{Timing attack}

In this sort of attack, the attacker induces the information character by character, dependent upon the yield kind of real/false. In time-based ambushes, attacker displays a deferment by implanting an additional SLEEP (n) call into the inquiry and after that viewing if the site page was truly by $\mathrm{n}$ seconds.

\section{Union Queries}

SQL licenses two requests to be joined and returned as one result set. For example, SELECT col1, col2, col3 FROM table1 UNION SELECT col4, col5, and col6 FROM table2 will return one result set involving the delayed consequences of the two request using this framework, an attacker can trap the application 
into returning data from a table not exactly the same as the one that was arranged by the creator. The mixed inquiry is associated with the primary SQL request using the catchphrase UNION as a piece of demand to get information related to various tables from the application.

Original query: select acc-number from user_details where $\mathrm{u} \_$id $=500$

Injected query: select acc-number from user_details where $u_{-} i d=$ ' 500 ' union select pin from acc_details where u_id='500'

\section{Blind SQL injection attacks}

Attacker commonly tests for SQL implantation vulnerabilities by sending the data that would achieve the server to create an invalid SQL question. If the server at that point restores a slip-up message to the client, the attacker will attempt to make sense of portions of the primary SQL request using information grabbed from these bungle messages.

\section{B. Aho-Corasick Algorithm}

In programming building, the Aho- Corasick figuring is a string looking estimation envisioned by Alfred V. Aho and Margaret J. Corasick. It is a kind of word reference matching estimation that discovers parts of a constrained course of action of strings (the "dictionary") inside a data content. It organizes all strings in the meantime. The multifaceted idea of the count is straight in the length of the strings notwithstanding the length of the looked content notwithstanding the number of yield matches. Observe that since all matches are found, there can be a quadratic number of matches if every substring matches (e.g. word reference $=$ an, aa, aaa, aaaa and input string is aaaa).

Calmly, the estimation builds up a restricted state machine that brings after a tire with additional associations between the diverse inside center points. These extra internal associations allow speedy moves between failed string matches (e.g. a sweep for cat in a tire that does not contain cat, yet rather contains truck, and along these lines would miss the mark at the center point prefixed by ca), to various branches of the tire that offer a run of the mill prefix (e.g., in the past case, a branch for trademark might be the best parallel move). This allows the machine to move between string matches without the necessity for backtracking.

As soon as the string word reference is known early, (e.g. a PC disease database), the improvement of the system can be performed once detached and the joined machine set away for later use. For this circumstance, its run time is immediate in the length of the commitment notwithstanding the number of facilitated sections. The Aho- Corasick string matching estimation confined the preface of the primary UNIX charge fgrep.

\section{Illustration:}

For this situation, we will consider a vocabulary involving the going with words: $\{a, a b, b a b, b c, b c a, c, c a a\}$.

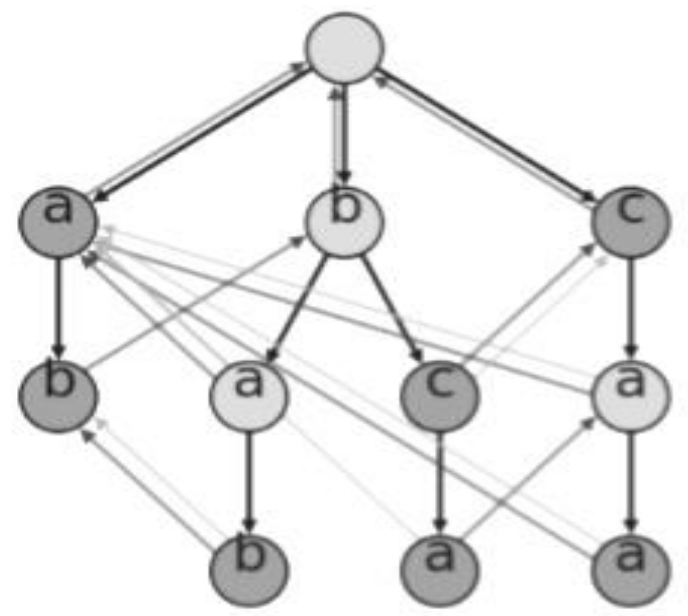

Figure 1.1 Aho- Corasick data structure created from the foreordained word reference 
The diagram underneath is the Aho- Corasick data structure created from the foreordained word reference, with each line in the table addressing a center point in the tire, with the fragmented way demonstrating the (stand-out) plan of characters from the root to the center point.

The data structure has one center point for each prefix of each string in the word reference. So if (bca) is in the vocabulary, at that point there will be center points for (bca), (bc), (b), and (). If a center is in the vocabulary then it is a blue center point. Else it is a diminish center point.

There is a dull composed "youth" bend from each center point to a center whose name is found by appending one character. So there is a dull bend from (bc) to (bca).

There is a blue composed "expansion" round fragment from each center point to the center that is the longest possible strict postfix of it in the graph. For example, for center point (caa), its strict postfixes are (aa) and (an) and (). The longest of these that exist in the chart is (a). So there is a blue roundabout fragment from (caa) to (a). The blue bends can be figured in straight time by on and on exploring the blue twists of a center's parent until the point that the intersection center point has a tyke matching the character of the goal center point.

There is a green "dictionary expansion" round section from each center to the accompanying center in the word reference that can be come to by taking after blue bends. For example, there is a green curve from (bca) to (an) in light of the way that (an) is the primary center point in the word reference (i.e. a blue center) that is accomplished when taking after the blue roundabout fragments to (ca) and subsequently on to (a). The green bends can be enrolled in coordinate time by more than once crossing blue round sections until a filled in center point is found, and remember this information.

At each movement, the present center is connected by finding its adolescent, and if that doesn't exist, finding its expansion's tyke, and if that doesn't work, finding its postfix's expansion's tyke, and so on, finally culmination in the root center point if nothing's watched some time as of late.

Right when the figuring accomplishes a center point, it yields all the word reference areas that end at the present character position in the data content. This is done by printing every center point came to by taking after the vocabulary expansion joins, starting from that center point, and continuing until the point that it accomplishes a center with no word reference postfix associate. In like manner, the center point itself is printed, in case it is a word reference area.

\section{PROPOSED SYSTEM}

In web security issues, SQLIA has the best by and large need. Basically, we can compose the territory and killing movement strategies into two general classes. Regardless approach is trying to perceive SQLIA through checking Anomalous SQL Query structure utilizing string matching, outline matching, and address managing. In the second approach utilizes information conditions among information things which are all the more unwilling to change for perceiving toxic database works out. In both, the classes, immense bits of the specialists proposed different game plans with joining information mining and interruption zone structures. Hal delicate et al built up a system that uses a model- based way to deal with overseeing recognize unlawful inquiries previously they are executed on the database. 
William et al proposed a structure WASP to check SQL Injection Attacks by a strategy called positive dirtying. Srivastava et al offered a weighted assembling tunneling approach for perceiving information base ambushes. The devotion of this paper is to propose a procedure for seeing and predicting SQLIA utilizing both static stage and component organize. The eccentricity SQL Queries are exposure in the static stage. In the dynamic stage, if any of the demand is seen as irregularity question then the new case will be delivered utilizing the SQL Query and it will be added to the Static Pattern List (SPL).

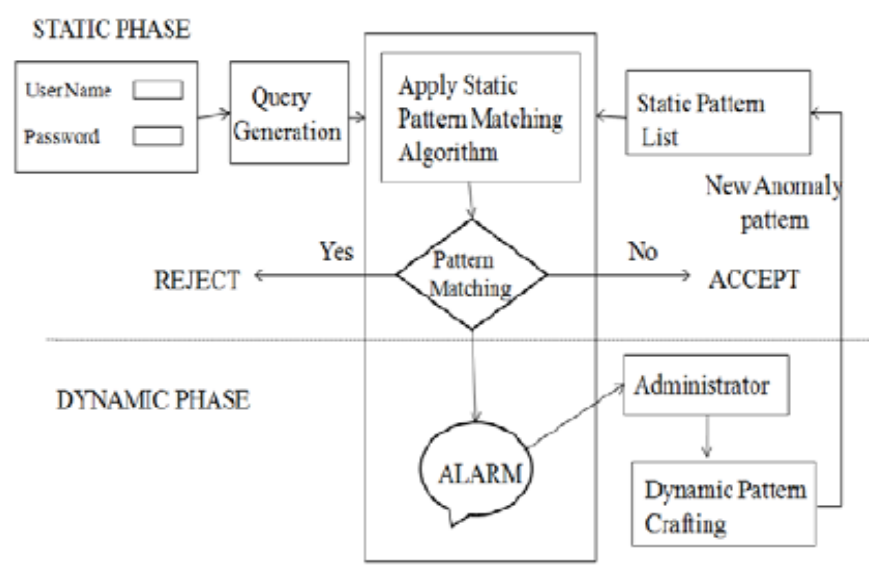

Figure 1.2 Architecture of SQLIA Detection

\section{CONCLUSIONS}

In this paper, we have proposed a scheme for the detection and prevention of SQL Injection Attack. We thought a strategy for insistence and slaughtering development of SQL Injection Attack (SQLIA) using Aho- Corasick configuration matching figuring. The system is completely robotized and perceives SQLIAs utilizing a model-based approach that hardens static and segment examination. This application can be utilized with different databases.

\section{REFERENCES}

[1]. M. A. Prabakar, M. KarthiKeyan, K. Marimuthu, "An Efficient Technique for Preventing SQL Injection Attack Using Pattern Matching Algorithm", IEEE Int. Conf. on Emerging Trends in Computing, Communication, and Nanotechnology, 2013.

[2]. E. Bertino, A. Kamra, and J. Early, "Profiling Database Application to Detect SQL Injection Attacks", In the Proceedings of 2007 IEEE International Performance, Computing, and Communications Conference, 2007.

[3]. G. T. Buehrer, B. W. Weide, and P. A. G. Sivilotti, "Using Parse Tree Validation to Prevent SQL Injection Attacks", Computer Science and Engineering, The Ohio State University Columbus, 2005.

[4]. J. H. Saltzer, M. D. Schroeder, "The Protection of Information in Computer Systems", In Proceedings of the IEEE, 2005.

[5]. Kamra, E. Bertino, and G. Lebanon, "Mechanisms for Database Intrusion Detection and Response", in the Proceedings of the 2nd SIGMOD Ph.D. Workshop on Innovative Database Research, 2008.

[6]. S. Axelsson, "Intrusion detection systems: A survey and taxonomy", Technical Report, Chalmers University, 2000.

[7]. S. F. Yusufovna, "Integrating Intrusion Detection System and Data Mining", IEEE Ubiquitous Multimedia Computing, 2008.

[8]. W. G. J. Halfond and A. Orso, "AMNESIA: Analysis and Monitoring for Neutralizing SQL Injection Attacks", College of Computing, Georgia Institute of Technology, 2005.

[9]. W. G. J. Halfond and A. Orso, "Combining Static Analysis and Runtime Monitoring to Counter SQL Injection Attacks", College of Computing, Georgia Institute of Technology, 2005. 
[10]. W. G. J. Halfond, A. Orso, and P. Manolios, "Using Positive Tainting and Syntax-Aware Evaluation to Counter SQL Injection Attacks", Proceedings of the 14th ACM SIGSOFT international symposium on Foundations of software engineering, 2006.

[11]. V. Aho and Margaret J. Corasick, "Efficient string matching: An aid to bibliographic search", Communications of the ACM, 1975.

\section{Cite this article as :}

Prof. Sweta Raut, Akshay Nikhare, Yogesh Punde, Snehal Manerao, Shubham Choudhary, "A Review on Methods for Prevention of SQL Injection Attack", International Journal of Scientific Research in Science and Technology (IJSRST), Online ISSN : 2395-602X, Print ISSN : 2395-6011, Volume 6 Issue 2, pp. 463-470, March-April 2019. Available at doi : https://doi.org/10.32628/IJSRST196258 Journal URL : http://ijsrst.com/IJSRST196258 